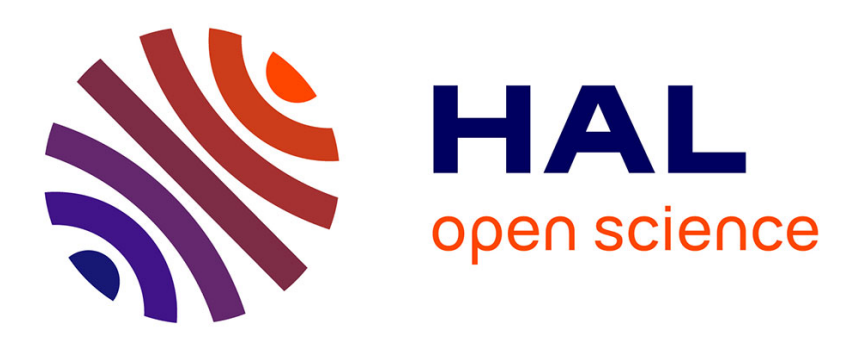

\title{
On the physical consistency of evolution laws obtained with sparse regression
}

Diego Matos Silva Lopes, Americo Cunha Jr

\section{To cite this version:}

Diego Matos Silva Lopes, Americo Cunha Jr. On the physical consistency of evolution laws obtained with sparse regression. W. Lacarbonara; B. Balachandran; M. J. Leamy; J. Ma; J. A. Tenreiro Machado; G. Stepan. Advances in Nonlinear Dynamics. NODYCON Conference Proceedings Series, Springer, Cham, pp.463-473, 2022, 10.1007/978-3-030-81166-2_41 . hal-03130833

\section{HAL Id: hal-03130833 \\ https://hal.science/hal-03130833}

Submitted on 3 Feb 2021

HAL is a multi-disciplinary open access archive for the deposit and dissemination of scientific research documents, whether they are published or not. The documents may come from teaching and research institutions in France or abroad, or from public or private research centers.

$$
\text { Copyright }
$$

L'archive ouverte pluridisciplinaire HAL, est destinée au dépôt et à la diffusion de documents scientifiques de niveau recherche, publiés ou non, émanant des établissements d'enseignement et de recherche français ou étrangers, des laboratoires publics ou privés. 


\title{
On the physical consistency of evolution laws obtained with sparse regression
}

\author{
Diego Matos Silva Lopes and Americo Cunha Jr \\ Rio de Janeiro State University - UERJ, Rio de Janeiro, Brazil \\ diego.matos@uerj.br, americo.cunha@uerj.br
}

\begin{abstract}
This work employs the technique known as sparse identification of nonlinear dynamics (SINDy) to infer, from a set of information provided by a given time-series, the evolution law of a dynamical system of interest, accessing the physical consistency of the obtained dynamic model. The Duffing oscillator is used as a benchmark due to the variety and richness of its dynamical behavior. The numerical experiments attempt to identify whether the method is capable of recognizing the correct evolution law and respecting basic principles of physics such as the balance of momentum and energy. The numerical results illustrate the method's ability to obtain approximations to the system evolution law that provides physically consistent behavior for short time intervals.
\end{abstract}

Keywords: nonlinear dynamics, data-driven dynamical system, sparse regression, SINDy, physics-informed machine learning

\section{Introduction}

Most classical dynamic systems, such as a harmonic oscillator, a simple pendulum have their evolution law inferred through the first principles $[1,2]$. However, some modern dynamical systems such as those that appear in areas like epidemiological modeling [3,4], neuroscience [5-7], have the basic evolution laws not well understood or even unknown so that the use of first principles to obtain the governing equations is unfeasible. In other applications, structural health monitoring $[8,9]$, for instance, it may be possible that the basic principles of dynamics are well understood, but some peculiarities not, which make it difficult to construct high-fidelity predictive models.

In this context, discover the evolution law for this kind of dynamical system in an analytical way becomes an almost impossible task. However, with the emergence of the information age, where data is exponentially generated and stored, it is natural to try to use information embedded in datasets associated with the dynamical system of interest to infer its underlying evolution law.

Neural networks (NN) [10-12] have a high capacity to learn complex patterns, which makes them at first glance natural candidates to be used in the search for mathematical expressions that represent the desired evolution law. However, despite having incredible power of prediction (interpolation) in the 
domain spanned by the training data, several NN architectures lack in providing interpretable expressions for the identified dynamic model, which makes it difficult to generalize (extrapolate) system behavior for unknown conditions.

Alternatively, regression techniques are more proper for providing the interpretability that NN does not have, which makes them an attractive tool to extract dynamic evolution laws from datasets [13]. In this scenario, stands out the technique known as sparse identification of nonlinear dynamical systems (SINDy) [13-16], a sparse regression method to identify evolution equations from data that proven to be efficient in different areas and problems, and stands out for three aspects: (i) interpretability of the obtained equation; (ii) excellent generalization (extrapolation) capability; (iii) computational efficiency. As several dynamic systems have evolution laws with only a few terms, SINDy looks for a sparse and parsimonious differential equation that best fits the known data.

If the method is applied in a system where the first principles are unknown, it is interesting to have some guarantee that the identified evolution law respects fundamental physical laws since their violation can translate into a dynamic behavior that is divergent from the original one. For instance, if the balance of momentum is not respected, mass or velocity are affected, potentially changing the inertial effects. When the energy balance is violated, the contribution from kinetic/potential energy to the mechanical energy can be in a proportion far from the original system, which can imply changes in the displacement/velocity. Even when a qualitatively good result is observed, small differences in the system parameters can significantly change quantitatively the long-term behavior.

This work aims to verify the physical consistency of the evolution law obtained by SINDy, checking if the identified dynamical system respects physical principles such as the balance of momentum and energy, and if the underlying balance of these quantities is an accurate approximation for those associated with the original system. For this purpose, the Duffing oscillator is used as a benchmark, where the underlying evolution equation is obtained from synthetic data that emulate experimental measurements for the displacement time-series of this dynamical system.

The rest of this manuscript is organized as follows. In section 2 , it is presented a brief explanation of the Duffing oscillator and the methodology used to identify the dynamic evolution laws. Some examples and test results are presented in section 3. Finally, in section 4 , the final remarks are highlighted.

\section{Methodology}

The dynamical system chosen to test the physical consistency of the identified dynamic laws obtained with SINDy is the Duffing oscillator, once this kind of oscillator has many possible applications, such as structural dynamics and energy harvesting [17-22], and a very rich and well known dynamic behavior. Fig. 1 shows a schematic of a vibratory system that behaves like a Duffing oscillator, in which the dynamic behavior evolves according to

$$
\ddot{x}+\delta \dot{x}+\alpha x+\beta x^{3}=\gamma \cos (\omega t),
$$


where the function $x=x(t)$ is the displacement of the beam tip, and the $\dot{x}$ and $\ddot{x}$ are respectively the first and second derivative of $x$, i.e., the velocity and acceleration [23]. The parameters in the equation of motion are the damping coefficient $\delta$; the linear stiffness $\alpha$; the nonlinear stiffness $\beta$; the external excitation amplitude $\gamma$; and the external excitation frequency $\omega$.

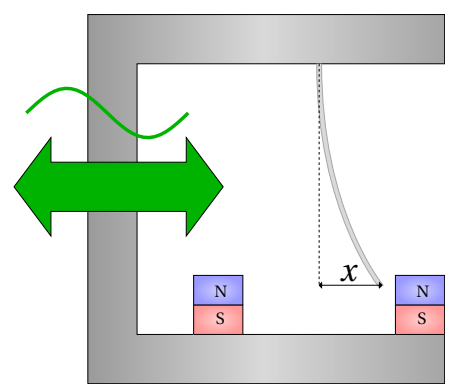

Fig. 1. Schematic of Duffing oscillator driven by a sinusoidal external excitation.

\subsection{Sparse identification of nonlinear dynamics}

The SINDy method can handle first order dynamical systems of form

$$
\dot{\mathbf{x}}=\mathbf{f}(\mathbf{x})
$$

where $\mathbf{x}$ is the state vector, $\dot{\mathbf{x}}$ is the time-derivative of the state vector, and $\mathbf{f}(\mathbf{x})$ is the unknown evolution law. Higher-order systems may be written as a first-order system by increasing the state dimension. For instance, the Duffing oscillator in Eq.(1) can be rewritten as

$$
\begin{aligned}
& \dot{x}_{1}=\phi x_{2}, \\
& \dot{x}_{2}=-\delta x_{2}-\alpha x_{1}-\beta x_{1}{ }^{3}+\gamma \cos x_{3}, \\
& \dot{x}_{3}=\omega .
\end{aligned}
$$

To determine the dynamic evolution law $\mathbf{f}$ it is necessary to have observations (time-series) of the system state $\mathbf{x}$ and velocity $\dot{\mathbf{x}}$, which are organized as follows

$$
\begin{array}{r}
\mathbf{X}=\left[\begin{array}{c}
\mathbf{x}^{T}\left(t_{1}\right) \\
\mathbf{x}^{T}\left(t_{2}\right) \\
\vdots \\
\mathbf{x}^{T}\left(t_{m}\right)
\end{array}\right]=\left[\begin{array}{cccc}
x_{1}\left(t_{1}\right) & x_{2}\left(t_{1}\right) & \ldots & x_{n}\left(t_{1}\right) \\
x_{1}\left(t_{2}\right) & x_{2}\left(t_{2}\right) & \ldots & x_{n}\left(t_{2}\right) \\
\vdots & \vdots & \ddots & \vdots \\
x_{1}\left(t_{m}\right) & x_{2}\left(t_{m}\right) & \ldots & x_{n}\left(t_{m}\right)
\end{array}\right], \\
\dot{\mathbf{X}}=\left[\begin{array}{c}
\dot{\mathbf{x}}^{T}\left(t_{1}\right) \\
\dot{\mathbf{x}}^{T}\left(t_{2}\right) \\
\vdots \\
\dot{\mathbf{x}}^{T}\left(t_{m}\right)
\end{array}\right]=\left[\begin{array}{cccc}
\dot{x}_{1}\left(t_{1}\right) & \dot{x}_{2}\left(t_{1}\right) & \ldots & \dot{x}_{n}\left(t_{1}\right) \\
\dot{x}_{1}\left(t_{2}\right) & \dot{x}_{2}\left(t_{2}\right) & \ldots & \dot{x}_{n}\left(t_{2}\right) \\
\vdots & \vdots & \ddots & \vdots \\
\dot{x}_{1}\left(t_{m}\right) & \dot{x}_{2}\left(t_{m}\right) & \ldots & \dot{x}_{n}\left(t_{m}\right)
\end{array}\right] .
\end{array}
$$


After having the data arranged in a matrix form, it is possible to build a library (dictionary) of candidate functions $\Theta(\mathbf{X})$ to construct the evolution law terms. In general, this library includes polynomials, trigonometric, exponential, logarithmic functions (among other possibilities), i.e.,

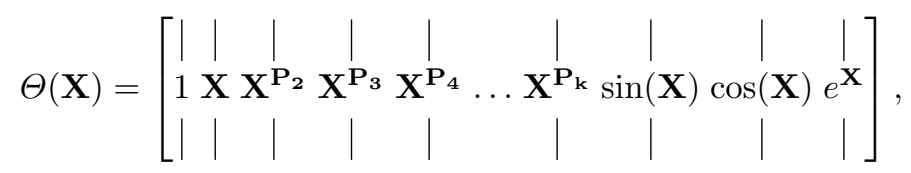

where each column in this matrix represents a candidate function, the $\mathbf{X}^{\mathbf{P}_{\mathbf{n}}}$ notation indicates all possible $n$-order polynomials formed by combining the state variables.

What SINDy does is to solve the following regression problem

$$
\dot{\mathbf{X}} \approx \Theta(\mathbf{X}) \Xi
$$

where the coefficient matrix $\Xi$ is determined by minimizing a misfit function that measures the discrepancy between $\Theta(\mathbf{X}) \Xi$ and $\dot{\mathbf{X}}$, i.e.,

$$
\Xi^{*}=\underset{\Xi}{\arg \min }\|\dot{\mathbf{X}}-\Theta(\mathbf{X}) \Xi\|_{2}+\lambda\|\Xi\|_{1}
$$

where $\|\cdot\|_{2}$ and $\|\cdot\|_{1}$ denote the norm-2 and norm-1, respectively.

Note that, to produce a sparse solution, a user-controlled $\lambda$ parameter is introduced, which acts as a threshold. A first least-squares regression results in a vector with estimated values for $\Xi$. Coefficients that represent the exact evolution law have more meaningful value than those that are not. After that, the absolute values of the coefficient vectors $\Xi$, which is smaller than a $\lambda$, have their values changed to zero. This process, known as sequential thresholded leastsquares, is repeated until a parsimonious evolution law is discovered [13]. For systems where the best parameter $\lambda$ is unknown, machine learning techniques for model validation are necessary to ensure a good result, e.g., cross-validation, among others [24].

\subsection{Training dataset}

In a typical scenario, the dataset used to infer the evolution law of the dynamic system are observations obtained with some type of sensor, in the field or in the laboratory. To emulate a set of displacement and velocity measurements for the Duffing oscillator, synthetic data are considered in this work. The corresponding time-series are generated with the aid of the numerical integrator ODE45 from MATLAB, fixing the time step as $\Delta t=0.01$. Concretely, a dataset with 151 equally spaced samples between 0 and 15 seconds is obtained with the numerical integrator and polluted with a zero-mean Gaussian white noise (with variance $\left.\eta^{2}\right)$ to emulate the fluctuations in experimental measurements. 


\subsection{Balance of energy and momentum}

The total energy, for both the original and the identified system, is the sum of the kinetic and potential energy

$$
E=\frac{1}{2} x_{2}^{2}+\frac{1}{2} \alpha x_{1}^{2}+\frac{1}{4} \beta x_{1}^{4} .
$$

Although this scalar quantity is not conserved, as the considered dynamics is dissipative, the balance between the mechanical energy of the system and the work done by external and dissipation forces must be respected, i.e.,

$$
\left[\frac{1}{2} x_{2}^{2}+\frac{1}{2} \alpha x_{1}^{2}+\frac{1}{4} \beta x_{1}^{4}\right]_{0}^{t}=\int_{0}^{t}\left[-\delta x_{2}^{2}(\tau)+\gamma \cos (\omega \tau) x_{2}(\tau)\right] d \tau .
$$

Similarly, the linear momentum variation has to balance the impulse of the forces acting on the system

$$
x_{2}(t)-x_{2}(0)=\int_{0}^{t}\left[-\delta x_{2}(\tau)-\alpha x_{1}(\tau)-\beta x_{1}^{3}(\tau)+\gamma \cos (\omega \tau)\right] d \tau .
$$

Therefore, if SINDy identifies the correct candidate functions, the balance of energy and momentum is respected by construction. However, even in this case, a question persists: Compared to the original dynamics, how representative (in quantitative terms) are these balance principles?

\section{Results and discussion}

Two parameter settings are used to evaluate the balance of energy and momentum in the SINDy method, nominally, the unforced conservative case, and the forced dissipative dynamics. For all the numerical experiments done and the database used, SINDy obtained enough parsimonious results to activate just the right candidate functions.

In the first case, the unforced conservative dynamics, 151 equally spaced time-samples are used in the training step, with a library of polynomial functions up to third-order without trigonometrical terms, with $\lambda=0.2$, and initial conditions $x_{0}=2$ and $v_{0}=-2$. Table 1 shows the values that SINDy inferred for two different noise intensities. The more noise, the less similar is the identified dynamics to their original counterpart.

In Fig. 2 the reader can see a verification test that estimates if the identified dynamics present an energy/momentum balance that is compatible with the invariances intrinsic to the original dynamics. The right side of Eqs.(10) and (11) are computed for both the identified dynamics and the original dynamics, and the differences between the respective quantities are computed, to estimate the balance error due to deviations in the identified coefficients. It is possible to

notice that the error in the momentum balance is directly proportional to the noise intensity, emphasizing the importance of obtaining good quality data. 
Table 1. Parameters of the original and identified dynamics in the conservative unforced case, with noise intensities equal to $\eta=0.001$ and $\eta=0.010$.

\begin{tabular}{lcccccc}
\hline & $\delta$ & $\alpha$ & $\beta$ & $\gamma$ & $\omega$ & $\phi$ \\
\hline Original value & 0.0 & 1.0 & -1.0 & 0.0 & 0.0 & 1.0 \\
Identified value $(\eta=0.001)$ & 0.0 & 0.9998 & -0.9999 & 0.0 & 0.0 & 1.0000 \\
Identified value $(\eta=0.010)$ & 0.0 & 0.9976 & -0.9993 & 0.0 & 0.0 & 1.0002 \\
\hline
\end{tabular}
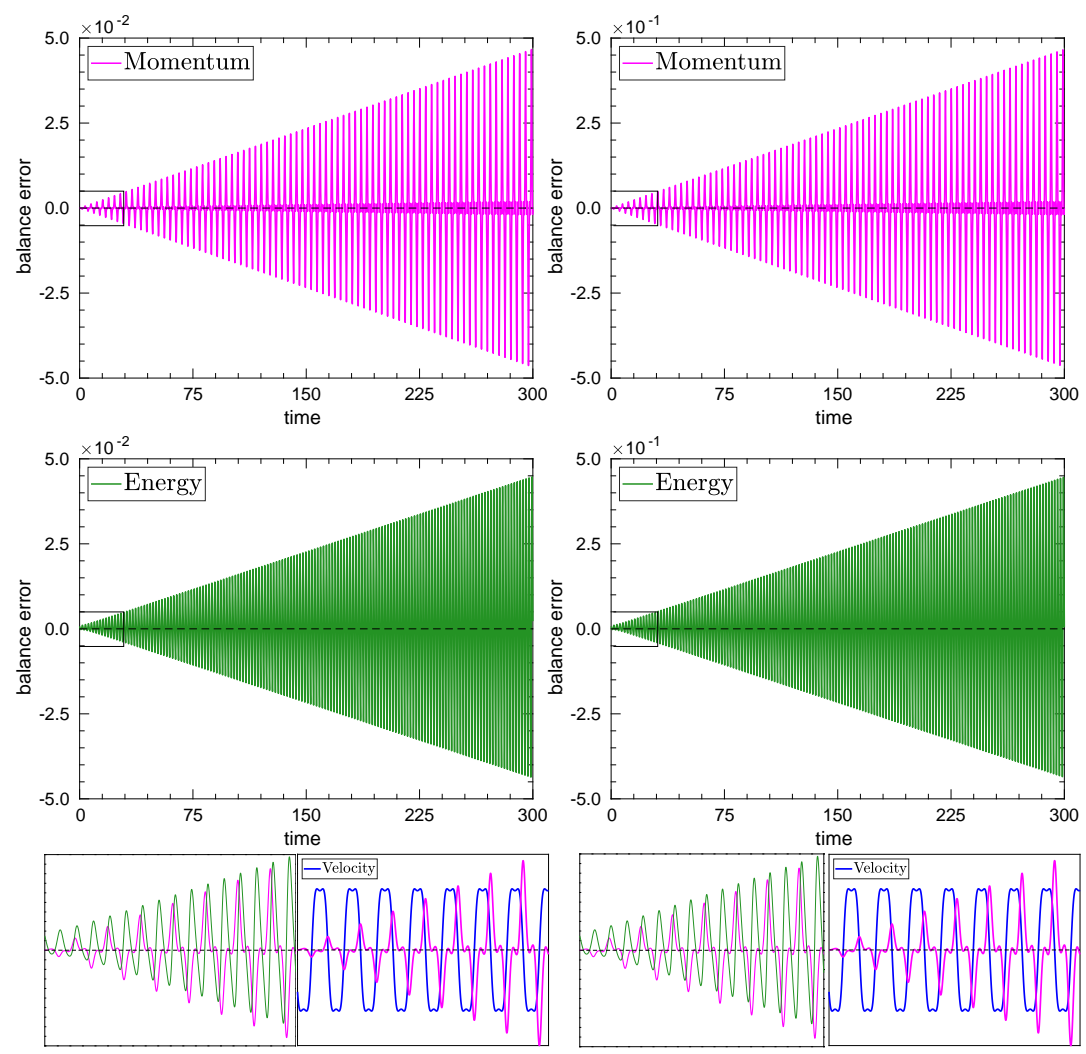

Fig. 2. The difference in the balance of energy and momentum between the original and identified dynamics for the unforced conservative case, with $\eta=0.001$ (left) and $\eta=0.010$ (right). The two insets below each graph show a zoom from the upper rectangles, where it is possible to see the symmetrical format of the curve. The velocity is plotted together with the momentum error, to show that the error in the momentum is a local maximum whenever the oscillator velocity is zero.

Therefore, despite identifying candidate functions correctly and with coefficients close to the original, the difference in both balances is a sinusoidal growing function over time. For the two noise intensities, the coefficients identified for the linear stiffness are lower than the original, while the nonlinear stiffness is 
slightly higher. Both systems after a long time become out of phase with the original one. For the systems with $\eta=0.010$, this is achieved early than the case where $\eta=0.001$, with time for that bigger than 300 .

In Fig. 3 a case without noise is considered, to assess the fundamental limit of noiseless measures. Note that, for different initial conditions, the error in the momentum balance is comparable to floating point noise, so that for any practical effect, SINDy can be considered to respect the linear momentum balance.
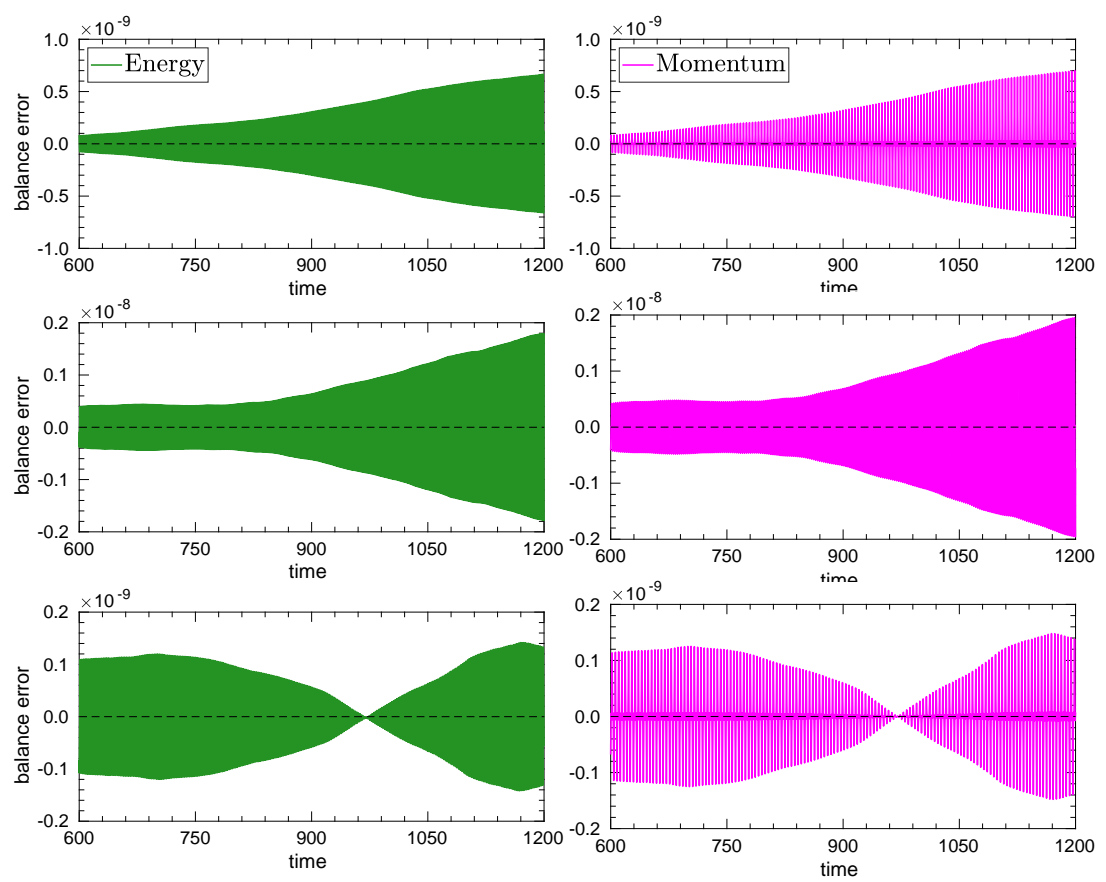

Fig. 3. The difference in the balance of energy and momentum between the original and identified dynamics with noise-free data, for different initial conditions. On the top, the initial conditions are $x_{0}=2$ and $v_{0}=-2$, on the middle $x_{0}=3$ and $v_{0}=2$, and for last, on the bottom $x_{0}=1$ and $v_{0}=2.5$.

Now the dissipative forced Duffing oscillator is considered. Two different noise intensities, $\eta=0.001$ and $\eta=0.010$, are employed once again, as well as the library of polynomial functions up to third-order with trigonometrical terms, 151 equally spaced time-samples, and $\lambda=0.02$, initial conditions $x_{0}=2$ and $v_{0}=-2$. The SINDy is able to identify the correct terms with great accuracy, despite the small amount of data, as can be seen in Table 2, which shows the system parameters for the original and the identified dynamics.

The balance of energy and momentum are plotted for this second case in Fig. 4, where it is possible to notice a richer oscillatory pattern, for both the momentum and energy errors, when compared to the first case. Looking at the 
energy error, initially, the local pikes and valleys are coinciding with those from the momentum error curve, but after some time, they become out of phase.

Table 2. Parameters of the original and identified dynamics in the dissipative forced case, with noise intensities equal to $\eta=0.001$ and $\eta=0.010$.

\begin{tabular}{lcccccc}
\hline & $\delta$ & $\alpha$ & $\beta$ & $\gamma$ & $\omega$ & $\phi$ \\
\hline Original value & -0.1 & 1.0 & -1.0 & 1.0 & 2.0 & 1.0 \\
Identified value $(\eta=0.001)$ & -0.0999 & 1.0001 & -1.0000 & 0.9998 & 2.0000 & 1.0000 \\
Identified value $(\eta=0.010)$ & -0.0992 & 1.0006 & -1.0001 & 0.9976 & 1.9996 & 1.0003 \\
\hline
\end{tabular}
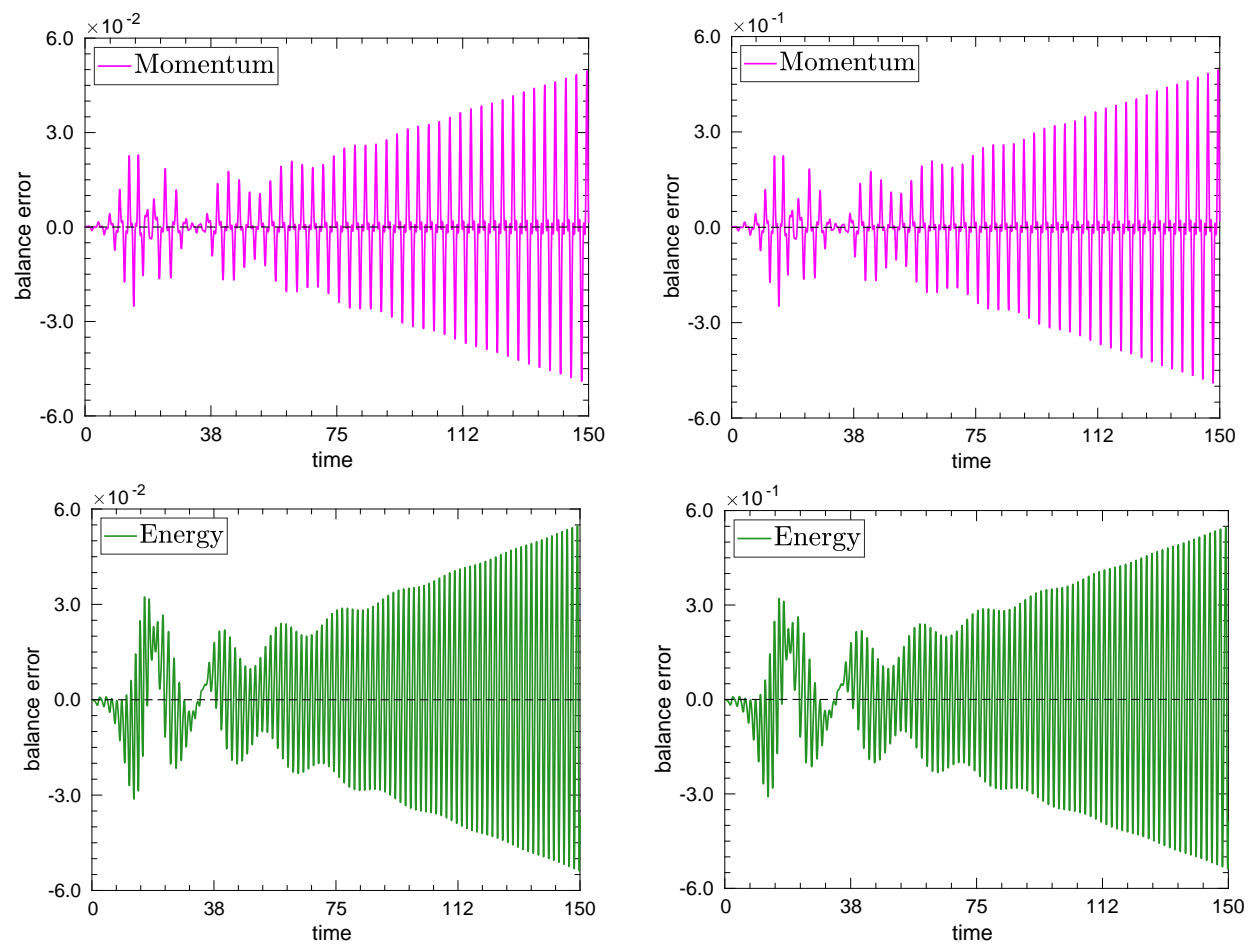

Fig. 4. The difference in the balance of energy and momentum between the original and identified dynamics for the dissipative forced case, with $\eta=0.001$ (left) and $\eta=0.010$ (right).

The difference in the balance of momentum and energy have both the same asymptotic behavior as in the conservative case, but now it is possible to observe an oscillatory pattern that is completely different from the conservative unforced 
case. A transient is noticed that is quickly dampened giving rise to a periodic stationary response, with an amplitude that increases (very slowly) with time.

\section{Conclusion}

This work presented a study on the physical consistency of the evolution law inferred from a given dataset employing the SINDy method. Two configurations of the Duffing oscillator are used to investigate the difference in energy and momentum balance with respect to the original dynamics. In all cases, SINDy identified the right candidate functions, therefore the balance of energy and momentum is respected. However, analyzing the difference in the energy and momentum balance identified in relation to the original, that function is growing rapidly over time. This implies that for long time periods, the identified dynamic will be out of phase to the original.

\section{Acknowledgments}

The authors would like to thank the financial support given to this research by the Brazilian agencies Coordenação de Aperfeiçoamento de Pessoal de Nível Superior - Brasil (CAPES) - Finance Code 001, and the Carlos Chagas Filho Research Foundation of Rio de Janeiro State (FAPERJ) under the following grants: $211.304 / 2015,210.021 / 2018,210.167 / 2019$, and 211.037/2019.

\section{References}

1. Corben, H.C., Stehle, M.S.: Classical mechanics: 2nd edition. Dover, New York (1994)

2. Meirovitch, L.: Methods of analytical dynamics. Dover, New York (2003)

3. Dantas, E., Tosin, M., Cunha Jr, A.: Calibration of a SEIR-SEI epidemic model to describe the Zika virus outbreak in Brazil. Appl. Math. Comput. 338, 249-259 (2018). doi:10.1016/j.amc.2018.06.024

4. Dantas, E., Tosin, M., Cunha Jr, A.: An uncertainty quantification framework for a Zika virus epidemic model. Journal of Computational Interdisciplinary Sciences 10(2), 91-96 (2019). doi:10.6062/jcis.2019.10.02.0163

5. Marblestone, A.H., Wayne, G., Kording, K.P.: Toward an integration of deep learning and neuroscience. Front. Comput. Neurosci. 10, 94 (2016). doi:10.3389/fncom. 2016.00094

6. Glaser, J.I., Benjamin, A.S., Farhoodi, R., Kording, K.P.: The roles of supervised machine learning in systems neuroscience. Progress in Neurobiology 175, 126-137 (2019). doi:10.1016/j.pneurobio.2019.01.008

7. Richards, B.A., Lillicrap, T.P., Beaudoin, P.: A deep learning framework for neuroscience. Nat. Neurosci. 22, 1761-1770 (2019). doi:10.1038/s41593-019-0520-2

8. Villani, L.G.G., Silva, S., Cunha Jr, A.: Damage detection in uncertain nonlinear systems based on stochastic Volterra series. Mech Syst Signal Pr. 125, 288-310 (2019). doi:10.1016/j.ymssp.2018.07.028 
9. Villani, L.G.G., Silva, S., Cunha Jr, A., Todd, M.T.: Damage detection in an uncertain nonlinear beam based on stochastic Volterra series: An experimental application. Mech Syst Signal Pr. 128, 463-478 (2019). doi:10.1016/j.ymssp.2019.03.045

10. Hasssanibesheli, F., Boers, N., Kurths, J.: Reconstructing complex system dynamics from time series: a method comparison. New J. Phys. 22, 073053 (2020). doi:10.1088/1367-2630/ab9ce5

11. LeCun, Y., Bengio, Y., Hinton, G.: Deep learning. Nature 521, 436-444 (2015). doi:10.1038/nature14539

12. Zhang, D., Guo, L., Karniadakis, G.E.: Learning in modal space: solving timedependent stochastic PDEs using physics-informed neural networks. SIAM J. Sci. Comput. 42(2), A639-A665 (2020). doi:10.1137/19M1260141

13. Brunton, S.L., Proctor, J.L., Kutz, J.N.: Discovering governing equations from data by sparse identification of nonlinear dynamical systems. PNAS 113(15), 3932-3937 (2016). doi:10.1073/pnas.1517384113

14. Kaiser, E., Kutz, J.N., Brunton, S.L.: Sparse identification of nonlinear dynamics for model predictive control in the low-data limit. Proc. R. Soc. A.474 (2018). doi: 10.1098/rspa.2018.0335

15. Bhadriraju, B., Bangi, M.S.F., Narasingam, A., Kwon, J.S.I.: Operable adaptive sparse identification of systems: Application to chemical processes. AIChE J. 66:e16980 (2020). doi:10.1002/aic.16980

16. Zhang, L., Schaeffer, H.: On the convergence of the SINDy algorithm. Multiscale Model. Simul., 17(3), 948-972 (2019). doi:10.1137/18M1189828

17. Cottone, F., Gammaitone, L., Vocca, H.: Nonlinear energy harvesting. Phys. Rev. Lett. 102(8), 080601 (2009). doi:10.1103/PhysRevLett.102.080601

18. Lopes, V.G., Peterson, J.V.L.L., Cunha Jr, A.: Numerical study of parameters influence over the dynamics of a piezo-magneto-elastic energy haversting device. In: XXXVII Congresso Nacional de Matemática Aplicada e Computaciona, Proceeding Series of the Brazilian Society of computational and Applied Mathematics, v.6, n.1, S.J. dos Campos-SP, Brazil (2017). doi:10.5540/03.2018.006.01.0407

19. Lopes, V.G., Peterson, J.V.L.L., Cunha Jr, A.: Nonlinear characterization of a bistable energy harvester dynamical system. In: Belhaq, M., (eds) Topics in Nonlinear Mechanics and Physics. Springer Proceedings in Physics, 228. Springer, Singapore (2019). doi:10.1007/978-981-13-9463-8_3

20. Peterson, J.V.L.L., Lopes, V.G., Cunha Jr, A.: Maximization of the electricalpower generated by a piezo-magneto-elastic energy harvesting device. In: XXXVI Congresso Nacional de Matemtica Aplicada e Computacional, Proceeding Series of the Brazilian Society of Computational and Applied Mathematics, v.5, n.1, GramadoRS, Brazil (2016). doi:10.5540/03.2017.005.01.0200

21. Guyomar, D., Richard, C., Badel, A., Lefeuvre, E., Lallart, M.: Energy Harvesting using Non-linear Techniques. In: Priya, S., Inman, D.J., (eds) Energy harvesting technologies. Springer, Boston, MA (2009). doi:10.1007/978-0-387-76464-1_8

22. De la Roca, L., Peterson, J.V.L.L., Pereira, M., Cunha Jr, A.: Control of chaos via OGY method on a bistable energy harvester. In: 25th ABCM International Congress on Mechanical Engineering, Uberlândia-MG, Brazil (2019).

23. Brennan, M.J., Kovacic, I.: Examples of physical systems described by the Duffing equation. In: Kovacic, I., Brennan, M.J., (eds) The Duffing Equation: Nonlinear Oscillators and Their Behaviour. Wiley (2011)

24. Bishop, C.M.: Pattern recognition and machine learning. Springer-Verlag, Berlim (2006) 